\title{
Women's perceptions of pharmacist-prescribed hormonal contraception
}

\author{
Ashley H. Meredith Pharm.D., FCCP ${ }^{1,2}$ ( ) | Nicole L. Olenik Pharm.D. ${ }^{1,3}$ | \\ Karen B. Farris Ph.D., MPA ${ }^{4}$ | Karen Suchanek Hudmon Dr.P.H., M.S. ${ }^{1}$
}

${ }^{1}$ Department of Pharmacy Practice, Purdue University College of Pharmacy, Indianapolis, Indiana

${ }^{2}$ Eskenazi Health, Indianapolis, Indiana

${ }^{3}$ Mathes Pharmacy, New Albany, Indiana

${ }^{4}$ Department of Clinical Pharmacy, University of Michigan College of Pharmacy, Ann Arbor, Michigan

\section{Correspondence}

Ashley H. Meredith, Department of Pharmacy Practice, Purdue University College of Pharmacy, 640 Eskenazi Avenue, Fifth Third Bank FOB, 3rd Floor, Indianapolis, Indiana 46202.

Email: ahmeredith@purdue.edu

Funding information

Purdue University

\begin{abstract}
Introduction: Unintended pregnancy is a public health issue, with young women in their late teens and early 20s at highest risk. Multiple approaches to increasing contraception access have been implemented, including adoption of statewide pharmacist prescribing legislation in multiple states. Indiana does not currently have such legislation in place. The objective of this study was to identify how women at risk for unintended pregnancy in central Indiana perceive pharmacist prescribing of contraception.
\end{abstract}

Study Design: Qualitative, one-on-one semi-structured interviews.

Methods: Purposive, convenience sampling of eligible women 18 to 29-years-old who live in Indiana was used. Recruitment occurred via printed flyers placed in public locations that were not focused on health care, free classified online advertisements, and electronic flyers within Facebook social groups relevant to the study population. Interviews were conducted via telephone until thematic saturation was achieved. Participant demographics, baseline characteristics, current and past barriers to obtaining contraception, and perception of a pharmacist contraception prescribing model were collected. Interview transcripts were coded in an iterative manner with qualitative data analysis software (MAXQDA, Version 12) until consensus was achieved.

Results: Fourteen women completed the study. Four primary themes emerged encompassing accessibility, communication, pharmacist attributes, and payment. Thirteen participants would personally utilize a pharmacist prescribing contraceptive service and all stated that this service would be beneficial for other women. Of those women expressing a preference $(n=7)$, a female pharmacist would be preferred for service delivery. Participants expressed concerns related to access by minors, skills and training of the pharmacist, and environment within the pharmacy.

Conclusions: Women in Indiana desire broader access to contraception. Efforts should be made at the individual pharmacist-provider level and statewide to explore strategies to increase access. Resources should be allocated to the creation of protocols for pharmacist prescribing within Indiana and other states.

\section{KEYWORDS}

contraception, perception, pharmacists, qualitative evaluation 


\section{1 | INTRODUCTION}

Because unintended pregnancy is a public health crisis, it is a target of the Healthy People 2020 (HP2020) initiative. ${ }^{1}$ Many of the Family Planning objectives of HP2020 aim to decrease unintended pregnancy through improved access to contraception. Young women in their late teens and early 20s exhibit the highest rates of unintended pregnancy; of these, $91 \%$ of pregnancies are among adolescents, and 59\% of pregnancies for women age 20-24 years are unwanted or mistimed. ${ }^{2}$ However, when barriers to access to same-day contraception are removed, unplanned pregnancy rates decrease by $20 \%-40 \%$. $^{3,4}$

One approach to increasing access to contraception is to provide it where adolescents and young women are already shopping, such as a community pharmacy within the county in which they reside. ${ }^{5}$ It has been shown that more than half of women visit a pharmacy at least once per month. In many states, expansion of the pharmacist prescribing model has been recognized by policy-makers through statewide legislation, and this could provide an additional method for enhancing access. $^{6-8}$ Overall, perceptions of pharmacist prescribing for contraception is positive-numerous studies have shown that most women, community pharmacists, student pharmacists, and other health care providers are supportive of this expanded role. ${ }^{9-14}$

The basic process for obtaining contraception via this mechanism is:

1. A patient presents to the community pharmacy, completes a selfadministered questionnaire to screen for medical contraindications, and her blood pressure is assessed.

2. A pharmacist with special training recommends appropriate contraceptive(s) based on responses to the questionnaire, patient preferences, and local statutes. If the patient is ineligible for a pharmacist prescribed method, or if she requests a product that is unavailable in the pharmacy, the pharmacist refers the patient to an appropriate provider for further evaluation.

3. Once a contraceptive has been selected, the pharmacist prescribes the medication and provides counseling that includes instructions for initiation, adherence, management of side effects, and expectations for follow-up visits.

Within Indiana, the rate of unintended pregnancy reached $49 \%$ in 2010 (55 000 pregnancies), resulting in an estimated 35200 births (64\%), 11000 abortions (20\%), and 8800 miscarriages (16\%). ${ }^{15}$ Statewide legislation allowing pharmacists to prescribe contraceptive medications does not currently exist in Indiana or in the majority of other states, however there is a broader form of pharmacist prescribing through collaborative drug therapy management (CDTM) that provides the framework for creating a similar model. CDTM is defined as "a collaborative practice agreement between one or more physicians and pharmacists wherein qualified pharmacists working within the context of a defined protocol are permitted to assume professional responsibility for a variety of functions, including patient assessment, ordering drug therapy-related laboratory tests, and selecting, initiating, monitoring, continuing, and adjusting drug regimens." ${ }^{16,17}$ As of 2016, 48 states including Indiana allow creation of CDTMs for the management of a variety of disease states (eg, diabetes or hypertension), making pharmacist prescribing of hormonal contraceptives possible. ${ }^{18}$ As a first step towards exploring this potential for an expanded role, the objective of this study was to identify how women at risk for unintended pregnancy in central Indiana perceive pharmacist prescribing as a method for obtaining contraception.

\section{2 | METHODS}

\section{1 | Design}

Participant interviews were conducted to gather information from women across central Indiana. One-on-one semi-structured interviews were completed and recorded, using an interview guide developed specifically for this study. Exempt status was received from the Human Research Protection Program at Purdue University.

\section{2 | Participant recruitment}

Purposive, convenience sampling was used to select participants residing within central Indiana. Central Indiana was selected as the recruitment site based on the large population of young women, ability to complete timely in-person interviews, and increased demographic diversity of potential participants. Eligible participants were females, aged 18 to 29-years-old, and able to read, speak, and understand English or Spanish. Women not meeting these inclusion criteria were excluded.

To facilitate recruitment, printed flyers were posted in public areas around central Indiana such as libraries, coffee shops, and community colleges. Flyers were not placed in locations focused on health care, such as pharmacies and clinics, in an attempt to recruit young women that may not routinely access health care services. In addition, a free classified advertisement was posted on Craigslist for Indiana and NUVO, a local independent alternative-newspaper, and electronic flyers were posted to various Facebook social groups relevant to the study population. Participants were recruited until thematic saturation was achieved. Saturation was monitored throughout recruitment and by the tenth interview, no new themes were identified. Four additional interviews had been scheduled prior to determining thematic saturation, therefore all participants were interviewed to confirm that no new themes were emerging.

\section{3 | Setting}

One of two investigators (AM, NO) completed the one-on-one interview with participants at a time that was mutually agreed upon between the investigator and participant. Participants were given the option of completing the approximately 30-minute interviews faceto-face or via telephone. 


\subsection{Data collection}

To ensure face validity of the question guide before completing interviews with study participants, each investigator completed at least two pilot interviews. At the beginning of each interview, investigators collected participant demographics (eg, age, race) and baseline characteristics (eg, current birth control use, past birth control use, current health conditions, health insurance status). The investigator then explored participants' current and past barriers to obtaining contraception, accessing their preferred pharmacy, experiences with their pharmacy and pharmacist, and perception of a pharmacist prescribing model for contraception (Supporting Information in Data S1). Participants could choose to skip any item that they were uncomfortable discussing. After obtaining participant permission, the investigators recorded all interviews. Recordings were transcribed verbatim using a Health Insurance Portability and Accountability Act (HIPAA)compliant service (Infraware, Terre Haute, Indiana) to ensure correct recall of statements, and to compile and de-identify information.

\section{5 | Data analysis}

To identify themes in participant responses, the interview transcripts were coded in an iterative manner through use of qualitative data analysis software (MAXQDA, Version 12, Berlin, Germany). Two investigators (AM, NO) independently coded all transcripts and discussed the rationale for any differences until consensus was achieved. If consensus could not be achieved, a third investigator was available to settle any discrepancies, but was not utilized. Recruitment closed once investigators agreed that thematic saturation had been attained.

\section{3 | RESULTS}

Fourteen women participated in the study; all opted for a telephone interview. On average, they were 25-years-old (range, 20-29), took
TABLE 1 Participant demographics

\begin{tabular}{|c|c|}
\hline Characteristic & $\mathrm{n}=14$ \\
\hline Age, mean (range) & 25 (20-29) \\
\hline \multicolumn{2}{|l|}{ Race } \\
\hline Black or African American, n (\%) & $1(7.1)$ \\
\hline White, n (\%) & $11(78.6)$ \\
\hline Prefer not to answer, n (\%) & $2(14.3)$ \\
\hline \multicolumn{2}{|l|}{ Ethnicity } \\
\hline Hispanic, n (\%) & $4(28.6)$ \\
\hline Non-Hispanic, n (\%) & $10(71.4)$ \\
\hline Current daily medications, mean (range) & $2(0-15)$ \\
\hline \multicolumn{2}{|l|}{ Health insurance for prescription medications } \\
\hline Yes, n (\%) & $11(78.6)$ \\
\hline No, $n(\%)$ & $3(21.4)$ \\
\hline \multicolumn{2}{|l|}{ Health insurance for birth control ${ }^{a}$} \\
\hline Yes, n (\%) & $8(61.5)$ \\
\hline No, n (\%) & $5(38.5)$ \\
\hline
\end{tabular}

${ }^{\mathrm{a}}$ Note: $\mathrm{n}=13$.

an average of two medications per day (range, 0-15), and six were currently using a hormonal contraceptive (Table 1; Figure 1).

Four primary themes emerged, encompassing accessibility, communication, pharmacist attributes, and payment. Each primary theme had three additional subthemes that were identified. Some themes and subthemes were predefined, while others emerged during analysis. Additional illustrative examples highlighting these themes and subthemes can be found in Table 2 .

\section{1 | Theme 1: Accessibility}

Within the theme of accessibility, many women commented about the current practice model of accessing a health care provider to obtain
FIGURE 1 Participant birth control use

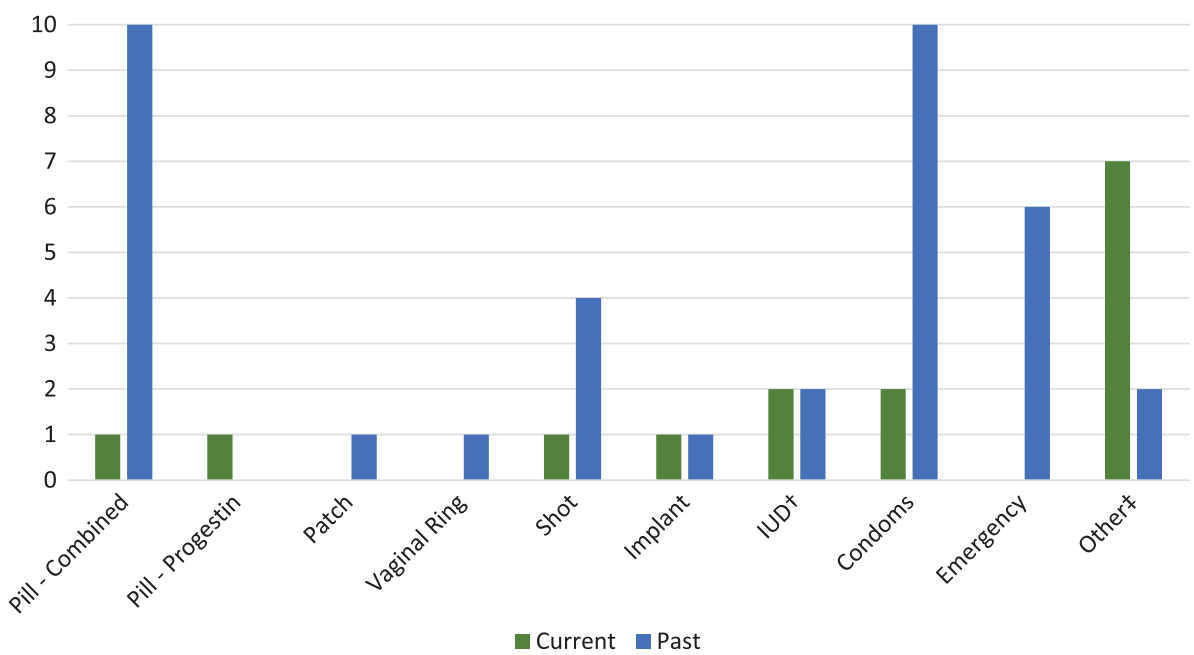

${ }^{\dagger}$ IUD (intrauterine device) includes hormonal and non-hormonal products. ¥Other methods include: natural family planning methods and abstinence. 
TAB LE 2 Themes and Representative Quotations

$\begin{array}{ll}\text { Primary theme } & \text { Subtheme } \\ \text { Accessibility } & \begin{array}{c}\text { Current practice } \\ \text { model }\end{array}\end{array}$

Wait times

Pharmacist

prescribing model

Parental consent

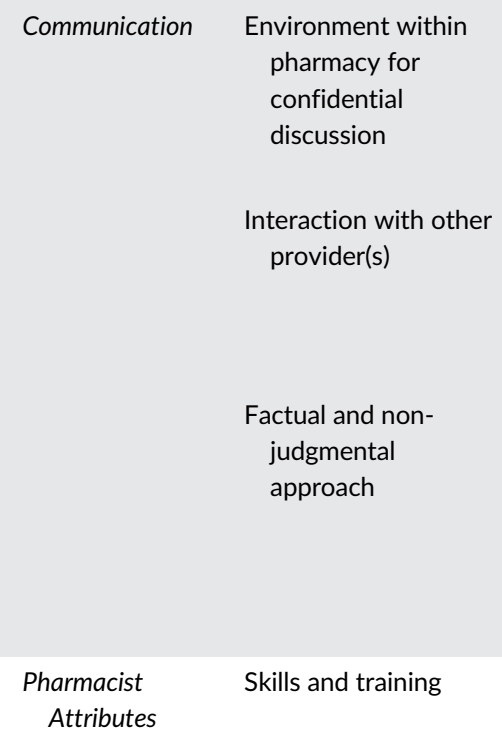

Personality and demeanor

\section{Quotations}

"I was just there for my six-week normal check. I didn't really want to be put on birth control right away because I kind of wanted my body to try to get back to normal before I got put back on it. My doctor strongly recommended it, so at that point in time they put me on the mini-pill or whatever. So I had to get checked by them and then I think I have a year supply. Then I will go back to get an exam again."Participant 8

"They have two separate offices, and I was scheduled at one and I showed up, and they were like, you are not scheduled for this office, so I had to drive all the way across town to a different office when I was told the opposite, so that was kind of a pain."-Participant 9

"Sometimes, like the prescription will be wrong and you have to wait for the OB to call you back, and a lot of phone tag"-Participant 7

"They [gynecologists] are really hard to get hold of. They don't really answer the phone that often and they don't tend to call you back, so it takes a while to get an appointment there."-Participant 9

"I honestly think especially in rural areas where there are not doctors, or not enough doctors, like where I live right now there are not enough family doctors, that service would probably be used a lot."Participant 5

"I think it is very convenient and I guess very convenient for other people that they have easier access to birth control as opposed to having to schedule an appointment with the physician and maybe wait if they weren't able to get in right away and it allows for access to that right away which would be helpful."-Participant 13

"I think one day 'What if my daughter has to use this?', like if it can be without a parent's knowledge, but I did that one time, you know?"-Participant 1

"A lot of young people might [not] want to go to the gynecologist necessarily, or they never have and so they are kind of nervous, but they still want the access to the birth control and they want to be safe, or they might need it for, who knows... a lot of reasons, so I think it would help young people because they would have better access to it and easier access to it."-Participant 9

"... in a little bit of a private secluded area so that they are not in a store that they frequent often, especially in case they were to see someone they knew or whatnot. So kind of just maybe in a remote area."-Participant 13

"Like a semi-closed off area where, you don't have other people listening to your conversation, because that's kind of a private, personal thing."-Participant 14

"I would prefer that [the pharmacist talk to other healthcare providers]. Then I think it would be safer if a doctor could review it and make the final call, at least knew about it ahead of time."-Participant 5

"Maybe some sort of release of information with your OB... that way they maybe could have access to medical records if they have any concerns or questions... or could review those types of things prior to maybe an appointment."-Participant 11

"As awkward and embarrassing as that is, they were pretty up front about it, and 'Scar tissue and stuff makes things uncomfortable, and having kids changes your anatomy' and stuff like that. So, they were like 'Well maybe you should look into the different birth control options if it is discomforting you."'Participant 7

"And someone who is professional and is willing to lay out all the pros and cons of different options and health risks of different options and someone that seems comfortable in what they are doing by providing you with birth control."-Participant 14

"Somebody with multiple health problems, the pharmacists generally aren't as knowledgeable about those, and it could cause a lot of interactions with the symptoms I currently have... my other health problems. And I feel like a pharmacist wouldn't be knowledgeable enough to address that and might prescribe something to somebody that could cause more harm than good."-Participant 5

"I guess the only hesitation would be the pharmacist isn't necessarily trained in that area, so I guess I would be a little hesitant about their knowledge base and their ability to discern between different kinds of birth control and what would be best for that. I don't really know what the separate qualifications are, from what I have heard it is mostly just personal preference between the different kinds of birth control. I would be concerned about the previous education of my pharmacist to make them qualified to make that decision."-Participant 12

"Probably one that is patient and has time to answer my questions if I should have them."-Participant 3 "Someone that is warm and friendly about it and very open and just patient and understanding, because, some people might be embarrassed or they just might be kind of timid to ask questions, so that would be, something to be like, 'Oh, okay', just that warm environment for that person to open up and be okay."-Participant 7 
TABLE 2 (Continued)

\begin{tabular}{|c|c|c|}
\hline \multirow[t]{2}{*}{ Primary theme } & Subtheme & Quotations \\
\hline & Sex & $\begin{array}{l}\text { "I would probably prefer a female, that is on birth control, just because they understand the side } \\
\text { effects a little bit more."-Participant } 6 \\
\text { "I think that it would be most comfortable for myself as well as other women to have a female } \\
\text { pharmacist doing this. I know I am a nurse and I know that I get that question a lot. It seems like } \\
\text { most females prefer when we do referrals to OB/GYN they prefer a female. So I think that maybe } \\
\text { that would put more people at ease or more women at ease when discussing different things that } \\
\text { there are concerns about and any issue getting birth control prescription from a pharmacist."- } \\
\text { Participant } 13\end{array}$ \\
\hline \multirow[t]{3}{*}{ Payment } & Insurance coverage & $\begin{array}{l}\text { "It would depend on how much it was, because there is that convenience factor, but it's like how much } \\
\text { are you willing to pay for the convenience factor. And it also depends would it just be a copay through } \\
\text { your insurance? Would the insurance cover it? Is it a non-insurance thing?"-Participant } 7 \\
\text { "Well if they were able to bill for it, that would obviously be better because they are providing a service, } \\
\text { but if they weren't, it would stay off my insurance and that would be a positive too."-Participant } 12\end{array}$ \\
\hline & Willingness to pay & $\begin{array}{l}\text { "I would expect that there would be some sort of cost involved. Yeah, I wouldn't think that it would be } \\
\text { free. I mean, it would be nice if it was free, but if there was a cost, that wouldn't discourage me from } \\
\text { using that."-Participant } 10 \\
\text { "You are paying for the convenience of it and you are paying for not having to go to your doctor. Your } \\
\text { doctor costs money anyway, it is just your insurance will sometimes cover it, but I would appreciate } \\
\text { the service so I wouldn't mind paying for it."-Participant } 12\end{array}$ \\
\hline & $\begin{array}{l}\text { Cost comparison to } \\
\text { provider visit }\end{array}$ & $\begin{array}{l}\text { "Well I mean if it was a small fee, I think that is... I mean they have to get paid too, so that wouldn't } \\
\text { bother me. I mean if it's going to be } \$ 80 \text { like it would be for an office visit, then I might as well just get } \\
\text { everything done at the same place. If it was like } \$ 15 \text { or } \$ 20 \text {, then I could see the value of it" - } \\
\text { Participant } 8 \\
\text { "I would probably not want to pay as much as I would pay for a doctor's appointment, but I understand } \\
\text { that it would be some of that pharmacist's time, so, you know, no such thing as a free lunch and all of } \\
\text { that. I am sure that I could understand there being some kind of cost, but since it would not be quite } \\
\text { as involved as an appointment with a medical doctor in an office with an exam, I would expect it to be } \\
\text { less."-Participant } 14\end{array}$ \\
\hline
\end{tabular}

Abbreviations. GYN, gynecologist; OB, obstetrician.

a new or refilled birth control prescription. Participants expressed frustrations with the need for scheduled appointments, required physical examinations, and other barriers such as obtaining childcare to attend visits.

"It's a little bit harder [to coordinate] because my daughter is autistic, so I have been trying to find a babysitter that knows how to handle some of the challenges that come with that. So, she did have to come with me, but they were pretty nice about the entire situation, they were very understanding, and she was relatively behaved that day, so... that helped."-Participant 7

A somewhat related, yet separate subtheme of wait times evolved throughout data analysis. Women were often frustrated with setting up and attending appointments, as well as receiving products from the pharmacy.

"And then also, the wait times... because when I was able to get my diaphragm in the past... there was a special order, like wait time, so that was also inconvenient. So yeah, child care for sure and then wait time for picking it up from the pharmacy."-Participant 14
When asked for their perceptions of a pharmacist prescribing model, 13 participants indicated they would personally utilize a pharmacist prescribing contraceptive service, and all participants stated that this service would be beneficial for other women. Many women perceived this service as convenient due to a lack of scheduled appointments and extended hours of availability, decreased waiting time, and increased health care resources.

"I would love that. I would love not having to call over and over again trying to get into my gynecologist every year. I also would skip the whole waiting process at the gynecologist. My pharmacy is closer than both my gynecologist's offices, so that would be positive. I don't... I'm young and I don't have any other health problems and I don't have a lot of concerns or things that I would need to talk to the gynecologist about, so it would just be... it would save me time and effort to go get something like that. I honestly... I would love that so much to just be able to go in there and talk to them and get a prescription that day."-Participant 9

Within accessibility, the idea of parental consent emerged. Some participants viewed this model as an option for young women that might not be comfortable disclosing to their parents that they are 
seeking birth control, while other participants expressed concerns that young women might be able to do just that.

"I feel like under the age of 16 they should have some sort of parental consent or at least have something like that or somebody over the age of 18 that they would have, but somebody that is there, involved in that, because it is a drug and I feel like parents should... or somebody... you know, even if it is an aunt or uncle or somebody that they trust that is not their parent, somebody should know the side effects and things like that and understand them, because I feel like even when I was taking it under 16, I didn't understand the side effects and things like that."-Participant 6

\section{2 | Theme 2: Communication}

Multiple concepts related to the primary theme of communication emerged throughout the interviews. A general concern raised by some participants was the environment within the pharmacy for a confidential discussion.

"Well I would like more of a secluded area talking about things, because I don't want the whole world [to] know I am on certain medications. So kind of doing it in a more secluded area versus just the consultation area that they have at the counter."-Participant 8

The way in which the pharmacist might have an interaction with other provider(s) was also discussed.

"I guess birth control can interact with a lot of different medications like antibiotics and stuff. I would want my other healthcare practitioners to know. The more people that know the more it becomes a privacy thing as well. So I guess I wouldn't mind them sharing the information because it is important to my health care, but I would just be concerned about the level of privacy my other health care physician has."-Participant 12

Participants discussed the factual and non-judgmental approach of a pharmacist in a positive way as it relates to discussing sensitive topics, however it was also viewed as a barrier to developing a more in-depth relationship.

"Then seeing a pharmacist you have a million things that you have to do at a time, at least from what it looks like. So whenever you ask a pharmacist questions most of the time, they are very cut to the point, because they have a million other things to be doing. So just having a rapport with that person I think would be helpful."-Participant 8

\section{3 | Theme 3: Pharmacist attributes}

Participants were asked a series of questions about pharmacists that would make them most comfortable utilizing this type of service. More often than not, participants voiced questions and concerns related to the skills and training of the pharmacist who would be performing this type of service, particularly if they had multiple chronic conditions or took multiple daily prescription medications.

"I think I would like if there were some type of continuing education or some type of training program. I guess if it is going to be implemented nationwide, it would be in the pharmacy school curriculum. Like for all the pharmacists that graduate before that, I think there should be some type of certification and just to get the information before they start doing this. I guess I would look for something like that. Like if you are newly out of school curriculum or someone who has gone back and like studied it all for the requirement."-Participant 12

The personality and demeanor of the pharmacist providing the service was also an important consideration.

"...and calming and understanding, they come off well educated regarding the several different types of birth control and if there are questions regarding different brands and side effects and things like that. The patient would be comfortable asking and knowing that they are being provided adequate information."Participant 13

The sex of the pharmacist was an important consideration for most women. Of those who expressed a preference $(n=7)$, a female pharmacist was preferred. The remaining participants did not mention the sex of the pharmacist as impacting their perceptions.

"I think that there's always, especially when you are seeing someone for birth control, it is sort of nice to have someone who is a woman... not that I wouldn't trust a man to do that, but I think women have the ability, even as doctors, to be more empathetic and, understand your concerns with a medication that a man may brush off as not being as strong of a concern."-Participant 14

\section{4 | Theme 4: Payment}

As a whole, participants expected to have an associated fee for pharmacist prescribing services, and this did not change their perceptions of the service. Participants were willing to pay an average of $\$ 50$ (range, \$20-\$100) for a pharmacist contraception-prescribing interaction. Many participants questioned whether insurance coverage would apply to this service. 
"It just depends on how much it costs. I think that insurance should be able to cover it if you have insurance."Participant 2

Regardless of whether insurance could be used, participants expressed an expectation of an associated fee and a willingness to pay for this service.

"I would think you would have to pay at least the minimal fee, that is an actual service they are providing, and most likely I would think extra training they have to get."Participant 5

The last aspect of payment for pharmacist prescribing that emerged was as a cost comparison to a provider visit. While participants were willing to pay a fee for this service, they also felt it should be more cost effective than a provider's office visit.

"I mean if it's $\$ 25$, that would be great. But if it is $\$ 50, I$ can go to my doctor for that."-Participant 1

\section{4 | DISCUSSION}

Legislation allowing pharmacists to prescribe contraceptive medications does not currently exist in Indiana or in the majority of other states, and currently in Indiana there is no legislation under consideration for pharmacists to prescribe contraceptives. However, CDTM laws make creation of a pharmacist contraceptiveprescribing model possible in the absence of such legislation. In considering how a pharmacist prescribing model using CDTM in Indiana would be implemented, the women in this study provided useful insight. The participants of this study perceived pharmacist prescribing as a more convenient model than the model they are currently utilizing to obtain contraception. As such, women are willing to pay for the convenience, which warrants further investigation in order to create a financially sustainable model. Another key aspect to implementation would be ensuring that women are aware of the training and qualifications of pharmacists to deliver a contraceptive-prescribing service, as this was a common question raised by participants. A structured training program would need to be utilized for any pharmacist wishing to adopt this model. Lastly, strong relationships with collaborating physicians would be beneficial for the success of pharmacist contraceptive-prescribing services. Many women expressed an increased comfort with pharmacist prescribing if their current provider was included in communication. Development of a CDTM protocol requires an overseeing provider that is willing to delegate responsibility to the pharmacist, which could lead to a mutually collaborative relationship that can serve to increase the comfort of women seeking services at the pharmacy.

The majority of women who participated in this study would personally utilize a pharmacist prescribing service for their contraceptive needs, and all participating women expressed that the service would be beneficial to others. Women are also willing to pay a fee for the convenience of this service, which could support financially viable business models. This is important, because pharmacists are not currently able to receive reimbursement from health insurance providers for this or other clinical services. While women expressed a willingness to pay an out-of-pocket fee, the amount was limited based on comparison to the cost of attending a traditional visit with their health care provider. While a patient's co-payment for their provider visit may be $\$ 50$, the actual cost of the visit is significantly higher. Discussions surrounding payment highlight the lack of understanding that many patients have surrounding the true cost of health care services and the complex reimbursement structure that exists.

As additional states continue to propose and pass pharmacist contraception-prescribing legislation or pharmacists create CDTM protocols for these medications, ${ }^{8,19}$ special consideration must be given to the age of women eligible to receive services. Multiple states currently restrict this service to women who are at least 18-years-old. ${ }^{20,21}$ However, women most at risk of unintended pregnancy are adolescents and young adults. ${ }^{2}$ Concerns related to parental consent were raised by participants of this study, as were potential positive experiences for young women. While not voiced during this study, the ability of adolescents to accurately recall their own medical history and independently consent to medical treatment is often a cited concern with the expansion of medical policy to young people. ${ }^{22}$ Ongoing research continues to demonstrate that adolescents have the ability and capacity to accurately self-screen for appropriate use of hormonal contraception. ${ }^{22-24}$ Additional research is needed, with consideration given for including this vulnerable population in any policies that enhance access to contraception.

Several limitations exist for this study. The perceptions expressed by participants might not be representative of those from women outside of central Indiana as the majority of participants were white, nonHispanic, and had health insurance. Perceptions of the health care system might vary from women who have different demographic characteristics. Recruitment methods could have potentially lead to bias in participation as women with strong opinions about contraception access may have been more likely to respond to study information posted in public areas.

\section{5 | CONCLUSIONS}

The results of this study suggest that women in central Indiana desire broader access to contraception that is convenient, safe, and delivered by a professional who makes them feel comfortable. Efforts should be made at the individual pharmacist-provider level and statewide to explore strategies to increase access to contraception, in line with the HP2020 goals and objectives. ${ }^{1}$ Resources should be allocated to the creation of CDTM protocols for pharmacist prescribing within Indiana, as well as in other states. 


\section{ACKNOWLEDGMENTS}

This research was granted exempt status by the institutional review board (IRB) at Purdue University. This research did not receive any specific grant from funding agencies in the public, commercial, or not-forprofit sectors. The authors would like to thank Nicholas Rattray for his assistance in initial project development and methodological design.

\section{CONFLICT OF INTEREST}

The authors declare no conflicts of interest.

\section{ORCID}

Ashley H. Meredith (DD https://orcid.org/0000-0001-6162-8043

\section{REFERENCES}

1. Office of Disease Prevention and Health Promotion. Healthy people 2020 [cited 2019 May 3]. Available from www.healthypeople.gov/ 2020/topics-objectives/topic/family-planning/objectives.

2. Secura GM, Madden T, McNicholas C, et al. Provision of no-cost, long-acting contraception and teenage pregnancy. N Engl J Med. 2014;371(14):1316-1323.

3. Colorado Department of Public Health and Environment. Taking the unintended out of pregnancy: Colorado's success with long-acting reversible contraception, 2017 [cited 2019 May 3]. Available from www.colorado.gov/pacific/cdphe/cfpi-report.

4. Birgisson NE, Zhao Q, Secura GM, Madden T, Peipert JF. Preventing unintended pregnancy: the contraceptive CHOICE project in review. J Womens Health. 2015;24(5):349-353.

5. Fakih S, Batra P, Gatny HH, Kusunoki Y, Barber JS, Farris KB. Young women's perceptions and experiences with contraception supply in community pharmacies. J Am Pharm Assoc. 2015;55(3): 255-264.

6. Association of State and Territorial Health Officials Staff. States explore bills to address contraceptive access in 2018 [cited 2019 May 3]. Available from www.astho.org/StatePublicHealth/ States-Explore-Bills-to-Address-Contraceptive-Access-in-2018/0315-18/.

7. Association of State and Territorial Health Officials. State initiatives to increase access to contraception [cited 2019 May 3]. Available from www.astho.org/Maternal-and-Child-Health/Increasing-Accessto-Contraception/Resources-Map/.

8. Free the Pill. What's the law in your state, 2019 [cited 2019 July 27]? Available from http://freethepill.org/statepolicies/.

9. Landau SC, Tapias MP, McGhee BT. Birth control within reach: a national survey on women's attitudes toward and interest in pharmacy access to hormonal contraception. Contraception. 2006;74(6): 463-470.

10. Foster DG, Landau SC, Monastersky N, et al. Pharmacy access to emergency contraception in California. Perspect Sex Reprod Health. 2006;38(1):46-52.
11. Feehan M, Durante R, Ruble J, Munger MA. Qualitative interviews regarding pharmacist prescribing in the community setting. Am J Health Syst Pharm. 2016;73(18):1456-1461.

12. Landau S, Besinque $\mathrm{K}$, Chung $\mathrm{F}$, et al. Pharmacist interest in and attitudes toward direct pharmacy access to hormonal contraception in the United States. J Am Pharm Assoc. 2009;49(1):43-50.

13. El-lbiary SY, Raine T, Mclntosh J, Darney PD, Harper CC. Pharmacy access to emergency contraception: Perspectives of pharmacists at a chain pharmacy in San Francisco. J Am Pharm Assoc. 2007;47(6):702-710.

14. Rafie S, Haycock M, Rafie S, Yen S, Harper CC. Direct pharmacy access to hormonal contraception: California physician and advanced practice clinician views. Contraception. 2012;86(6):687-693.

15. Frost JJ, Frohwirth L, Zolna MR. Contraceptive needs and services, 2014 update [cited 2019 May 3]. Available from www.guttmacher. org/report/contraceptive-needs-and-services-2014-update.

16. Hammond RW, Schwartz AH, Campbell MJ, et al. Collaborative drug therapy management by pharmacists-2003. Pharmacotherapy. 2003; 23(9):1210-1225.

17. American College of Clinical Pharmacy, SE MB, Dopp AL, et al. ACCP white paper: Collaborative drug therapy management and comprehensive medication management - 2015. Pharmacotherapy. 2015;35 (4):e39-e50.

18. Hill JD, Hill JM, Gentile NJ. A review of state pharmacist collaborative practice laws. Am J Health-Syst Pharm. 2016;73(18):1467-1472.

19. National Alliance of State Pharmacy Associations (NASPA). Pharmacist Prescribing for Homonal Contraceptive Medications, May 24, 2019. Available at: https://naspa.us/resource/contraceptives/. Accessed July 27, 2019.

20. Sarna KA, Vinson MN, Fink III JL. Prescriptive Authority for Pharmacist: Oral Contraceptives. Pharmacy Times. November 2018.

21. Mospan G. Hormonal contraceptive consent by minors: Implications for pharmacists. US Pharm. 2018;43(5):22-25.

22. Williams R, Hensel D, Lehmann A, Sarin T, Shew M, Ott M. Adolescent self-screening for contraindications to combined oral contraceptive pills. Contraception. 2015;92(4):380.

23. Wilkinson TA, Vincent AH, Vielott TL, Meagher CG, Ott MA. Expanding adolescent contraceptive access to pharmacies: Adolescent capacity to self-screen for contraindications (poster). Presented at the Pediatric Academic Societies meeting. Toronto, Canada: Pediatrics; 2018.

24. Wilkinson TA, Meredith AH, Vielott TL, Meagher CG, Ott MA. The role of chronic illness and birth control experience in adolescents capacity to self-screen for hormonal contraception (poster). Presented at the North American Forum on Family Planning meeting. New Orleans, Louisiana: Contraception; 2018.

\section{SUPPORTING INFORMATION}

Additional supporting information may be found online in the Supporting Information section at the end of this article.

How to cite this article: Meredith AH, Olenik NL, Farris KB, Hudmon KS. Women's perceptions of pharmacist-prescribed hormonal contraception. J Am Coll Clin Pharm. 2020;3: 425-432. https://doi.org/10.1002/jac5.1169 